\title{
Impact Evaluation of Changes in the Manufacturing Line of Cyproterone Acetate through Analysis of Comparative Dissolution Profile
}

\author{
By Terezinha de Jesus Andreoli Pinto* \\ Rogério Takao Okamoto ${ }^{\dagger}$ \\ Wesley Anderson de Oliveirat \\ Daniela Dal Molim Ghisleni ${ }^{\circ}$
}

\begin{abstract}
In recent years, FDA has placed more emphasis on a dissolution profile comparison in the area of post-approval changes and biowaivers. A dissolution profile comparison between pre-change and post-change products, or regarding different strengths, helps assure similarity in product performance and signals bioinequivalence. This work aims to evaluate the impact caused by the change of the tablet compression machine in the manufacturing of $50 \mathrm{mg}$ cyproterone acetate tablets, by examining the comparative dissolution profile between different batches of the drug. For this purpose, the following method was used: the dissolution medium was composed of sodium dodecyl sulfate $0.07 \%$ in $0.01 \mathrm{M}$ hydrochloric acid, apparatus II (paddle), paddle rotation at 100 revolutions per minute, $900 \mathrm{~mL}$ volume of medium in each vessel, $37.5 \pm 0.5$ OC of medium temperature. Aliquots of $5.0 \mathrm{~mL}$ of sample were collected at 5, 10, 15, 20, 30 and 45 minutes. Then, the solutions of the sample and the standard (50.5 $\mu \mathrm{g} / \mathrm{mL})$ were evaluated using a spectrophotometer with wavelength adjusted to $285 \mathrm{~nm}$. From this the comparative dissolution profile was plotted considering the drug percentage dissolved for each batch in study as a function of the dissolution time. The two drug lots tested were manufactured in the same industry using different compression machines in the production line. Statistical analysis of data was performed and the similarity factor (f2) was calculated. For this study, 12 different tablet batches were used, in a total of 12 cyproterone acetate determinations per batch for each collected time reported. The results, calculated in the percentage of cyproterone dissolved in the medium, indicated that both lots of drugs tested showed values greater than $85 \%$ ( $Q>70 \%$ ) in the first 15 minutes of
\end{abstract}

\footnotetext{
*Professor, Sao Paulo University, Brazil.

${ }^{\dagger}$ Analyst, Sao Paulo University, Brazil.

*Analyst, Sao Paulo University, Brazil.

Analyst, Sao Paulo University, Brazil.
} 
Vol. 1, No. 2 Pinto et al: Impact Evaluation of Changes in the Manufacturing Line...

analysis. The mean final results (45 minutes) were equivalent to $107.73 \%(R S D=2.98 \%)$ and $108.58 \%(R S D=1.44 \%)$, respectively, independently of the changes on the compression machine used in the manufacturing line. The statistical analysis was used to evaluate result variability and to determine the similarity factor among the different batches of drugs tested ( $f 2=57.64)$, what allowed to conclude that the drugs tested showed similar dissolution profile. The results of dissolution efficiency calculation are: $83.14 \%$ $(R S D=1.89)$ to dissolution profiles obtained to the cyproterone tablets manufactured with compression machine 1 and $79.83 \%$ $(R S D=1.93)$ to the cyproterone tablets manufactured with compression machine 2. Statistical analysis showed that the differences observed between the results. The results based on the statistical calculations of $f 2$ showed equivalence in the dissolution profile, and different performances based on calculation of the dissolution efficiency (D.E.). However, the dissolution method could be reevaluated to analyze properly the product in study, fast dissolving formulation, using discriminative conditions. ${ }^{l}$ Key words: Dissolution profile, cyproterone acetate, similarity factor ( $f 2)$, efficiency dissolution (D.E.), dissolution test.

\section{Introduction}

The goal of tablet compression is to produce dosage forms that achieve the desired bioavailability and clinical effect [1]. Several authors studied the effect of the compaction process parameters on the granule properties. Other authors investigated the effect of the compaction force on tablet strength of a sustainedrelease tablet and found that the compaction force had a significant effect on the tablet hardness [2].

The absorption of a solid dosage form after oral administration depends on three factors: the release of the substance taken, the dissolution of the drug under physiological conditions and the permeability across the gastrointestinal tract. Due to the critical nature of the first two of these steps, an vitro dissolution may be relevant to the prediction of in vivo performance [3,4].

In vitro dissolution testing is an economic and useful quality control tool to effectively assure acceptable production of tablets, capsules and other solid dosage forms [5]. Dissolution is the process by which a solid solute enters a solution. In the pharmaceutical industry, it may be defined as the amount of drug substance that goes into the solution per unit time under standardized conditions of liquid/solid interface, temperature and solvent composition [6].

Dissolution testing is used as a quality control procedure in pharmaceutical production, in product development to assist in selection of a candidate formulation, in research to detect the influence of critical manufacturing

\footnotetext{
${ }^{1}$ The authors are grateful to São Paulo University (USP) and to CONFAR Laboratory.
} 
variables such as binder effect, mixing effect, granulation procedure, coating parameters, excipient type and/or in comparative studies of different formulations, in vitro-in vivo correlations and possibly as an in vivo surrogate under strictly defined conditions [7].

Cyproterone acetate is very soluble in dichloromethane. The free alcohol is an anti androgen, the acetate is both an anti-androgen and a progestagen [8]. The pharmaceutical dosage form is a progestational antiandrogen with potent antigonadotropic activity that results in rapid suppression of serum testosterone. Used as a single agent, cyproterone acetate yields a total androgen blockade. Cyproterone acetate may afford transient objective improvement in patients not responding to other forms of hormone deprivation. The drug may be used to suppress the hot flushes associated with orchiectomy. Cyproterone acetate induces local tumor regression; owing to its reversible effects, it is useful as neoadjuvant or adjuvant androgen withdrawal therapy in patients with lower-stage disease undergoing radical surgery or radiotherapy [9].

This work aims to evaluate the impact caused by the tablet compression machine change in the manufacturing of $50 \mathrm{mg}$ cyproterone acetate tablets by examining the comparative dissolution profile between different batches of the drug.

\section{Materials and Methods}

\section{Materials}

Sodium dodecyl sulfate (Synth, Brasil) and hydrochloric acid ACS (Merck, Germany).

To this study 24 tablets of the same lot of cyproterone acetate $50 \mathrm{mg}$ were used, having 12 of them been submitted to compression using a tablet compression machine, and the remaining 12 subjected to compression by another equipment.

\section{Dissolution Testing}

Dissolution tests were performed with a Ethinik Techonology (2996ATPS) dissolution system configured as USP-apparatus II, paddle rotation at 100 revolution per minute and $900 \mathrm{~mL}$ volume of medium in each vessel. The dissolution medium was composed of sodium dodecyl sulfate $0.07 \%$ in $0.01 \mathrm{M}$ hydrochloric acid, and the bath temperature was adjusted to $37.5 \pm 0.5{ }^{0} \mathrm{C}$.

Aliquots of $5.0 \mathrm{~mL}$ of sample were collected at 5, 10, 15, 20, 30 and 45 minutes. The amounts of drug dissolved were determined in $1 \mathrm{~cm}$ quartz cells, employing an Ultra-Violet/Visible Spectrophotometer, Thermo Scientific (Evolution/201). Determinations were carried out against a blank dissolution medium. Samples and standard solutions were prepared in medium dissolution to obtain $50 \mu \mathrm{g} / \mathrm{mL}$ concentration solutions. The dissolution test used is a pharmacopeial method [10] and was partially validated in our laboratory.

After the dissolution profile of 12 tablets from the first compression machine was evaluated, the other 12 tablets were analyzed in the same way. 
The percentage of the drug dissolved in the medium was calculated and the graphic of the percentage of drug released in the medium $\mathrm{x}$ time (minutes) was plotted.

\section{Calculations}

A changed product may also be a lower strength of a previously approved drug product. In the presence of certain minor changes, the single-point dissolution test may be adequate to ensure unchanged product quality and performance. For more major changes, a dissolution profile comparison performed under identical conditions for the product before and after the change(s) is recommended. Dissolution profiles may be considered similar by virtue of (1) overall profile similarity and (2) similarity at every dissolution sample time point. The dissolution profile comparison may be carried out usingl independent or dependent model methods [11].

For the analysis of the dissolution profiles, the similarity factor (f2) described in the FDA and EMEA guidelines was used [11, 12, 13]. The similarity factor (f 2 ) is a logarithmic reciprocal square root transformation of the sum 2 of squared error and is a measurement of the similarity in the percent $(\%)$ dissolution between the two curves:

$$
f_{2}=50 \times \log \left\{\left[1+\left(\frac{1}{n}\right) \times \sum_{i=1}^{n}\left(R_{f}-T_{t}\right)^{2}\right]^{-0,5} \times 100\right\}
$$

where $n$ is the number of time points, $R$ is the dissolution value of the reference $t$ (prechange) batch at time $t$, and $T$ is the dissolution value of the test (postchange) batch $t$ at time $t$ [11].

The dissolution efficiency (D.E.) was proposed by khan and Rhodes and is defined [14]:

$$
\text { D.E. }=\left(\int^{\mathrm{t} 2} \mathrm{t} 1 \mathrm{y} \cdot \mathrm{d}_{\mathrm{t}} / \mathrm{y}_{100} \cdot\left(\mathrm{t}_{2}-\mathrm{t}_{1}\right)\right) \times 100 \%
$$

Where: $y$ is the percentage of dissolved product. D.E. is then the area under the dissolution curve between time points $t_{1}$ and $t_{2}$ expressed as a percentage of the curve at maximum dissolution, $y_{100}$, over the same time period. It is preferable to choose a time interval corresponding to 70-90\% dissolution unless one wishes to compare an early part of the dissolution curve.

\section{Results and Discussion}

The percentage of cyproterone acetate dissolved in the dissolution medium was calculated for both situations evaluated in this work and the results are demonstrated in Table 1. 
Table 1. Percentage of Cyproterone Acetate dissolved in the Dissolution Medium using the Tablet Compression Machines 1 (Drug_A) and 2 (Drug_B)

\begin{tabular}{c|c|c|c|c|c|c|c}
\hline \multicolumn{7}{c}{ \% Drug Dissolved } \\
\hline \multicolumn{2}{c|}{ Time (minutes) } & 5 & 10 & 15 & 20 & 30 & 45 \\
\hline \multicolumn{2}{c|}{ Medium (mL) } & 900 & 900 & 900 & 900 & 900 & 900 \\
\hline \multirow{2}{*}{ Drug_A } & \begin{tabular}{c} 
\% Average \\
\cline { 2 - 8 }
\end{tabular} & 44.07 & 74.38 & 88.59 & 96.86 & 104.08 & 107.73 \\
\cline { 2 - 8 } & \% RSD & 17.25 & 6.75 & 4.80 & 4.02 & 2.65 & 2.98 \\
\hline \multirow{2}{*}{ Drug_B } & $\begin{array}{c}\text { \% } \\
\text { Average }\end{array}$ & 58.02 & 81.53 & 100.93 & 100.93 & 106.02 & 108.58 \\
\cline { 2 - 8 } & \% RSD & 11.28 & 5.86 & 2.29 & 2.29 & 1.76 & 1.44 \\
\hline
\end{tabular}

Average of \% cyproterone acetate dissolved obtained in dissolution profile (12 tablets)

The results indicate that the change in the line manufacturing resulted in an increase of the drug content percentage in the medium. The relative standard deviations calculated to the line manufacturing using the compressor machine $\mathrm{B}$ were lower than those observed using the compressor machine A.

The percentage of the drug dissolved in the medium was calculated and the graphic of the percentage of drug released in the medium $\mathrm{x}$ time (minutes) was plotted. The comparative dissolution profile of the drugs in study is shown in Figure 1. The profiles are well correlated although Drug_B dissolution was faster than that of Drug_A in the first minutes of the test.

Figure 1. Graphic of the Comparative Dissolution Profile to the Tablets Obtained with the Different Compression Machines

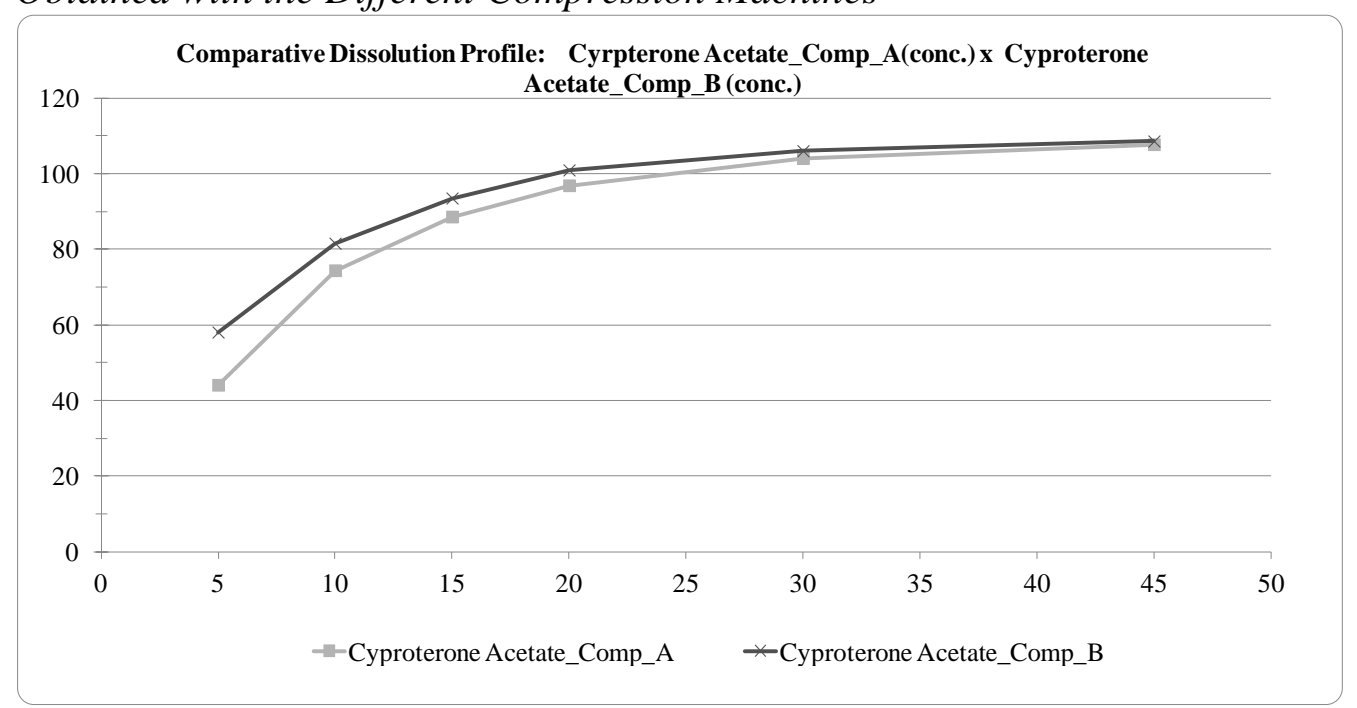

The similarity factor (f2) was calculated according to the official guidelines. The result (f2 $=57.64$ ) indicated the two drugs evaluated had similar dissolution profiles, regardless of the equipment used in the manufacturing line. The results obtained with the calculation of the dissolution efficiency (D.E.) are: $83.14 \%(\mathrm{RSD}=1.89)$ to dissolution profiles obtained to the cyproterone tablets manufactured with compression machine 1 and $79.83 \%$ $(\mathrm{RSD}=1.93)$ to the cyproterone tablets manufactured with compression 
machine 2. Statistical analysis showed that the differences observed between the results of the dissolution efficiency (D.E.) are significant to $95 \%$ confidence intervals $\left(\mathrm{t}_{\text {calc }}=4.24 \geq \mathrm{t}_{\text {crit }}=2.07\right)$. Based on these results it is possible to conclude that the dissolution method is not discriminative to the drug formulation used in this study, given that more than $85 \%$ of drug is dissolved in the dissolution medium in the first 15 minutes (fast dissolving drug). It might be useful to develop and validate a new dissolution method to this product.

\section{Conclusions}

Based on the similarity factor (f2) it is possible to conclude that the drugs tested have similar dissolution profiles independently of the tablet compressor machine used in the manufacturing line. The dissolution efficiency (D.E.) calculation demonstrated that the differences obtained to the manufacturing lines are significant. However, the dissolution method could be reevaluated to analyze properly the product in study using discriminative conditions.

\section{References}

1. Khan, K.A.; Rhodes, C.T. Effect of variation in compaction force on properties of six direct compression tablet formulations. Journal of Pharmaceutical Sciences. 65(12), 1835-1837, 1976.

2. Boniatti, J. et al. Galenic approaches in troubleshooting of glibenclamide tablet adhesion in compression machine punches. Saudi Pharmaceutical Journal (2013), http://dx.doi.org/10.1016/j.jsps.2013.08.002

3. Amidon, G.L.; Lennernas, H.; Shah, V.P.; Crison, J.R. A theoretical basis for a biopharmaceutic drug classification drug classification: the correlation of in vitro drug product dissolution and in vivo bioavailability. Pharm. Res., v.12, p.413420, 1995.

4. Lourenço, F.R.; Ghisleni, D.D.M.; Yamamoto, R.N.; Pinto, T.J.A. Comparison of dissolution profile of extended-release oral dosage forms - Two one-sided equivalence test. Brazilian Journal of Pharmaceutical Sciences, v. 49, n.2, apr./jun.,p. 367-371, 2013.

5. Maggio, R.M.; Castellano, P.M.; Kaufman, T. A new principal component analysis-based approach for testing "similarity" of drug dissolution profiles. European Journal of Pharmaceutical Sciences, v. 34, p. 66-77, 2008.

6. Lakshimi, C.S.; Badarinath, A.V. An updated review of dissolution apparatus for conventional and novel dosage forms. International Journal of Pharma Research \& review, 2(7), p. 42-53; 2013.

7. Pillary, V.; Fassihi, R. Evaluation and comparison of dissolution data derived from different modified release dosage forms: an alternative method. Journal of Controlled Release, 55 (1998) 45-55.

8. Merck Index: an encyclopedia of chemicals, drugs, and biologicals. 14.ed. Whitehouse Station: Merck Research Laboratories, 2006, p.2774.

9. Goldenberg, S.L.; Bruchovsky, N. Cyproterone acetate is a progestational antiandrogen with potent antigonadotropic activity that results in rapid 
suppression of serum testosterone. Used as a single agent, cyproterone acetate yields a total androgen blockade. Urol Clin North Am, Feb 18 (1), p. 11-22; 1991.

10. British Pharmacopoeia.- Cyproterone Tablets. Volume III, 2010, p.2603.

11. Food and Drug Administration .Guidance for Industry Dissolution Testing of Immediate Release Solid Oral Dosage Forms Additional, copies are available from: http://www.fda.gov/cder/guidance.htm. Access in 04/01/2014.

12. European Agency for the Evaluation of Medicinal Products. Note for Guidance on Quality of Modified Release Products: A. Oral Dosage Forms, B. Transdermal Dosage Forms; Section I (Quality), Unit H.M.E., EMEA, London, UK, 1999.

13. Haaser, M.; Naelapää, K.; Gordon, K.C.; Pepper, M.; Rantanen, J.; Strachan, C.J.; Taday, P.F.; Zeitler, A.; Rades, T. Evaluating the effect of coating equipment on tablet film quality using terahertz pulsed imaging. European Journal of Pharmaceutics Biophamaceutics 85(2013), p. 1095-1102.

14. Anderson, N.H.; Bauer, M.; Boussac, N.; Khan-Malek, R.; Munden, P.; Sardaro, M. An evaluation of in vitro dissolution profiles. Journal of Pharmaceutical and Biomedical Analysis 17, p.811-822, 1998. 
